\title{
Editorial for "Failed Total Hip Arthroplasty: Diagnostic Performance of Locoregional Lymphadenopathy at MRI to Identify Infected Implants"
}

T otal hip arthroplasty (THA) is a highly successful surgical intervention. ${ }^{1}$ The frequency of primary THA has grown steadily in recent years owing to a rapid increase in the prevalence of osteoarthritis, but so have the rates of revision surgery for failed THA. ${ }^{1,2}$ This is attributable to an increased life expectancy in an aging population and the fact that patients undergo this procedure at an ever younger age. ${ }^{3}$ Prevention of (re-)revision THA is of high importance because of the high risk of complications and costs. Multiple causes of failure have been identified, of which infection is one of the most devastating. In the case of periprosthetic joint infection (PJI), microbes tend to form a biofilm on implant surfaces, which renders antibiotic penetration difficult. Consequently, surgical intervention is often required consisting of retention of the implant and debridement, or one- or two-stage revision. ${ }^{1}$ In order to plan the most appropriate treatment strategy, PJI needs to be diagnosed (or excluded) before the surgery. Diagnosis of PJI remains challenging, however, as no "gold standard" exists and current clinical, laboratory, and imaging strategies have low sensitivity, especially in delayed, often low-grade infection with mostly nonspecific symptoms. ${ }^{4}$

Imaging might play an important role in detecting PJI, because of its noninvasive nature and novel methods that have the potential to increase diagnostic performance. Current imaging strategies include conventional imaging, computed tomography (CT), magnetic resonance imaging (MRI) and sometimes nuclear imaging methods, although the added value of the latter is not clear. ${ }^{5}$ Conventional imaging can detect signs of loosening, but it provides no information whether this has an aseptic or infectious cause. CT has the ability to detect bone defects, but is insensitive to soft-tissue abnormalities. MRI displays softtissue abnormalities with the best contrast and dedicated metal artifact reduction sequences (MARS) are particularly useful for diagnosing other causes of failure, such as metallosis. There is a lack of literature, however, on the diagnostic performance of MRI for PJI, especially in light of these new artifact reduction techniques.

In this issue of JMRI, an article by Albano et $\mathrm{al}^{6}$ advances our knowledge of the value of commonly used imaging features of THA failure, and of new indices derived from the number and size of locoregional lymph nodes detected on MRI in the identification of infected hip implants. ${ }^{6}$ This well-designed study included 119 patients with failed hip THA, who underwent first-time revision surgery. Prior to this, all patients underwent MRI with a MARS protocol on which a comprehensive set of MR features was assessed, including periprosthetic bone destruction, soft-tissue mass, effusion, synovial edema, lamellated synovitis, extracapsular edema, fibrous periprosthetic membrane, bone edema, and extracapsular collection/sinus tract. Moreover, the total numbers and ratios of affected lymph nodes compared to unaffected lymph nodes in several locoregional areas were defined, an interesting point of view, as enlarged lymph nodes are generally associated with infection, but have not been the focus of attention yet on MRI for hip protheses.

Albano et $\mathrm{al}^{6}$ showed relatively limited accuracy in the hip of the more established MRI features to diagnose PJI, with values ranging from about $50-80 \%$, in which synovitis and lamellated synovitis had the highest odds ratios. The novel lymph nodal indices showed accuracies ranging from $85 \%$ up to $93 \%$. The combination of lamellated synovitis with lymphadenopathy only slightly improved the accuracy, to $90-95 \%$.

In fact, when only lymph nodes are to be considered, one might stick to CT as a more practical and faster solution, as these are easily visible on CT with the contralateral side included in the field-of-view by default. Nevertheless, MRI delivers far more information on other potential failure causes. Future studies might focus on the difference in diagnostic performance between these two modalities. For now, this article emphasizes the importance of looking at the broader 
picture, including locoregional lymph nodes as a sign of infection, instead of only focusing on features surrounding the prothesis.

Rianne A. van der Heijden, MD, PhD $\mathbb{D}$ and

Edwin H.G Oei, MD, PhD

Department of Radiology \& Nuclear Medicine

Erasmus MC University Medical Center

Rotterdam, The Netherlands

E-mail: r.a.vanderheijden@erasmusmc.nl

\section{References}

1. Ferguson RJ, Palmer AJ, Taylor A, Porter ML, Malchau H, Glyn-Jones S. Hip replacement. Lancet 2018;392(10158):1662-1671.

2. Kurtz S, Ong K, Lau E, Mowat F, Halpern M. Projections of primary and revision hip and knee arthroplasty in the United States from 2005 to 2030. J Bone Joint Surg Am 2007;89(4):780-785.
3. Kurtz SM, Lau E, Ong K, Zhao K, Kelly M, Bozic KJ. Future young patient demand for primary and revision joint replacement: National projections from 2010 to 2030. Clin Orthop Relat Res 2009;467(10): 2606-2612.

4. Izakovicova P, Borens O, Trampuz A. Periprosthetic joint infection: Current concepts and outlook. EFORT Open Rev 2019;4(7): 482-494.

5. Palestro CJ, Love C. Role of nuclear medicine for diagnosing infection of recently implanted lower extremity arthroplasties. Semin Nucl Med 2017;47(6):630-638.

6. Albano D, Messina C, Azgra L, et al. Failed total hip arthroplasty: Diagnostic performance of locoregional lymphadenopathy at MRI to identify infected implants. J Magn Reson Imaging 2020; (this issue).

DOI: 10.1002/jmri.27333

Level of Evidence: 5

Technical Efficacy Stage: 2 